\title{
Intravenous leiomyomatosis of the uterus with extension to the right heart
}

\author{
Yu-Feng Lou', Xin-Ping Shi ${ }^{2}$ and Ze-Zhou Song ${ }^{3 *}$
}

\begin{abstract}
A 42-year-old woman admitted with debilitation and engorgement both lower extremities. Transthoracic twodimensional echocardiography, abdominal ultrasound and computerized tomography revealed a lobulated pelvic mass, a mass within right internal iliac vein, both common iliac vein, as well as the inferior vena cava, extending into the right atrium. In addition, echocardiography and abdominal ultrasound showed the tumor of right atrium and inferior vena cave has no stalk and has well-demarcated borders with the wall of right atrium and inferior vena cave. Hence, the presumptive diagnosis of IVL was made by echocardiography and abdominal ultrasound and the presumptive diagnosis of sarcoma with invasion in right internal iliac vein, both common iliac vein, the inferior vena cava, as well as the right atrium was made by multi-detector-row computerized tomography. The patient underwent a one-stage combined multidisciplinary thoraco-abdominal operation under general anaesthetic. Subsequently the pathologic report confirmed IVL.
\end{abstract}

\section{Introduction}

Benign smooth muscle neoplasms or leiomyomas are extremely common uterine tumors. On rare occasions, the neoplasms exhibit unusual growth patterns [1]. One such pattern is intravenous leiomyomatosis (IVL), which is an uncommon neoplasm characterized by intravascular proliferation of a histologically benign-looking smooth muscle cell tumor mass but not invading the tissue. Although IVL is usually confined to the pelvis and histologically benign, IVL exposed to venous blood that flows to the heart and may penetrate the inferior vena cava, reach the right heart chambers, or extend to the right pulmonary artery [2], which is named intracardiac leiomyomatosis and may results in cardiac symptoms and cardiac murmur, fainting and even in some cases, sudden death [3]. Because of rarity, IVL is occasionally misdiagnosed or diagnosed lately, and subsequently improperly treated and correct preoperative diagnosis of IVL depends on a huge index of doubt. Therefore, we present a case of IVL, which was cued by transthoracic echocardiography and abdominal ultrasound and confirmed by histopathological evaluation and review the literature.

\footnotetext{
* Correspondence: zezhou_song@126.com

${ }^{3}$ Department of Ultrasound, The First Affiliated Hospital, College of Medicine, Zhejiang University, Hangzhou, China

Full list of author information is available at the end of the article
}

\section{Case Report}

The patient was a 42-year-old woman admitted with debilitation and engorgement of both lower extremities. In past, she has undergone hysterectomy one year and six months ago because of hysteromyoma and was never found to have any heart diseases and relevant history. She was never found to have diabetes mellitus and denied relevant history of smoking. On examination, there was no abnormality in physical examination including cardiac auscultation etc. except for mild edema of both lower extremities. The laboratory examinations revealed normal results including tumor markers etc.

Transthoracic two-dimensional echocardiography showed normal parameters of the left and right ventricular wall thickness, size and function. The right atrium was mildly enlarged and filled with a medium echogenic oval tumor mass which is approximately $5.5 \mathrm{~cm}^{*} 2.3 \mathrm{~cm}$ (Figure 1). The tumor mass originated from inferior vena cave, extended into right atrium (Figure 2), moved back and forth through the tricuspid orifice into right ventricle. The tumor of right atrium and inferior vena cave has no stalk and has well-demarcated borders with the wall of right atrium and inferior vena cave (Figure 2), which caused the tumor wandering within right atrium and inferior vena cave. The moderate tricuspid regurgitation was detected. In addition, abdominal ultrasound revealed a pelvic mass and continued 


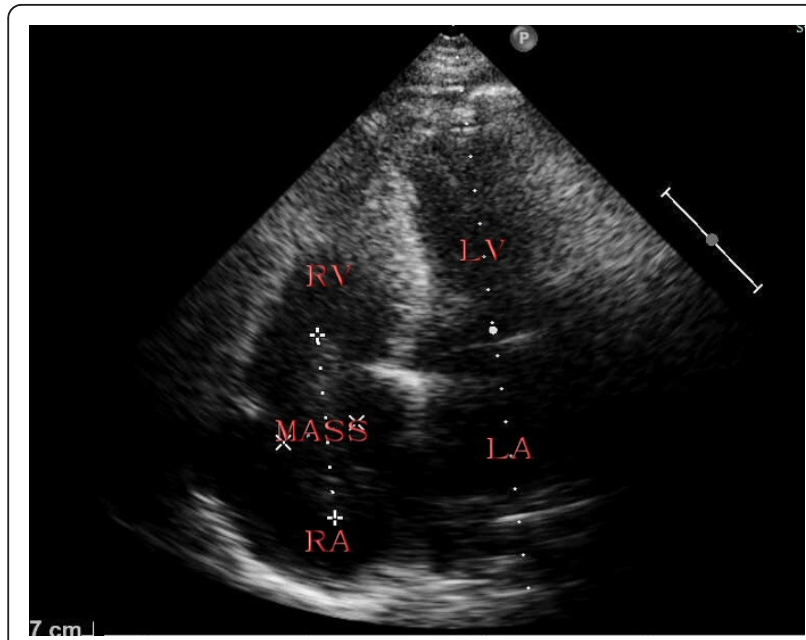

Figure 1 Apical four chamber view showing right atrium filled with a medium echogenic oval tumor mass by transthoracic two-dimensional echocardiography. RV: right ventricle, RA: right atrium, LV: left ventricle, LA: left atrium.

medium echogenic oval tumor mass within right internal iliac vein, both common iliac vein, as well as the inferior vena cava (Figure 3). Hence, the presumptive diagnosis of IVL was made by echocardiography and abdominal ultrasound.

The multi-detector-row spiral plain scan and contrastenhanced computerized tomography of thoracic cavity, abdominal cavity and pelvic cavity, which was clearly visualized in the present case, revealed a lobulated pelvic

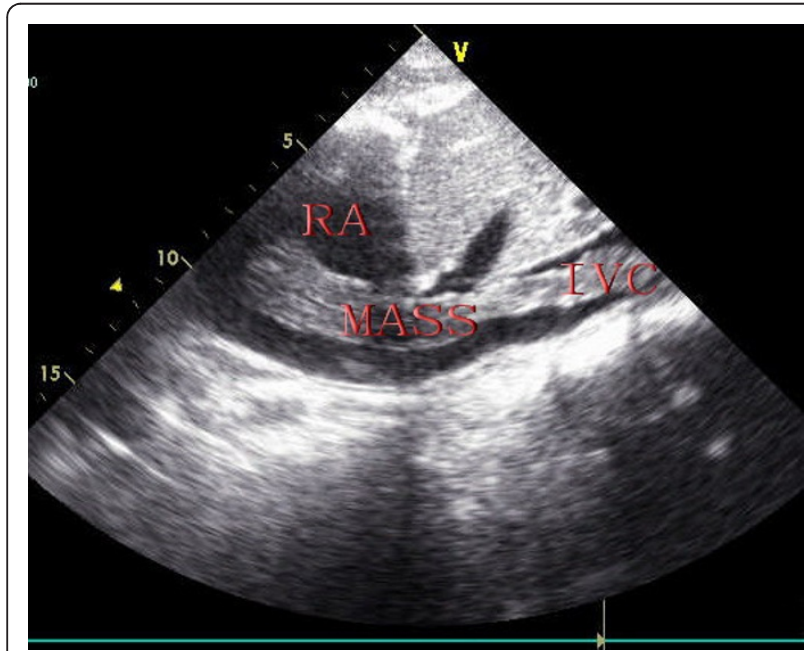

Figure 2 Under-ensisternum view showing mass originated from inferior vena cave, extended into right atrium and that mass of right atrium and inferior vena cave has no stalk and has well-demarcated borders with the wall of right atrium and inferior vena cave by transthoracic two-dimensional echocardiography. RA: right atrium, IVC: inferior vena cave.

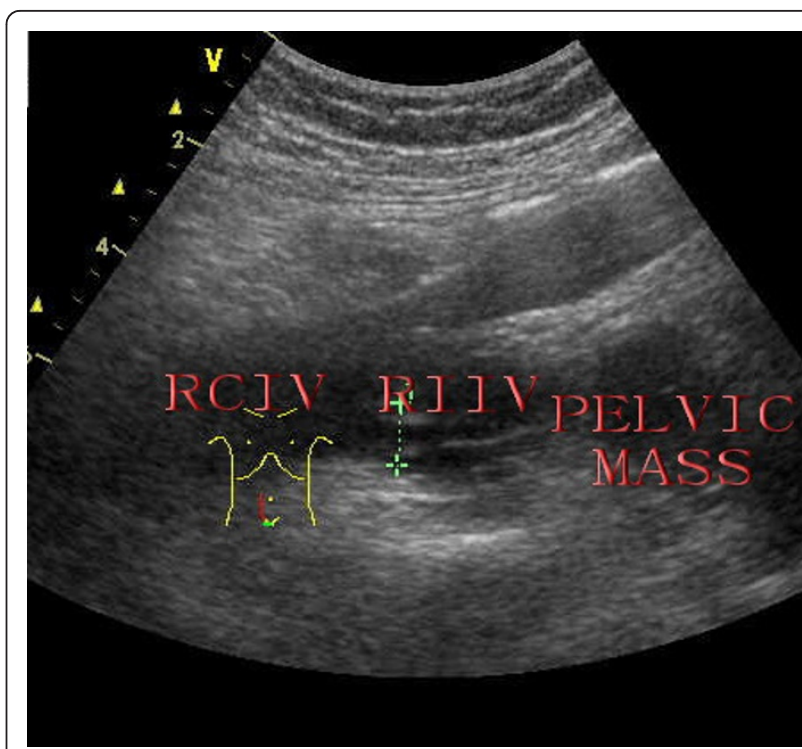

Figure 3 Pelvic view showing a pelvic mass and continued medium echogenic oval tumor mass within right internal iliac vein, right common iliac vein by abdominal ultrasound. RIIV: right internal iliac vein, RCIV: right common iliac vein.

mass, a low attenuation-filling continued defect was noted from an enlarged right internal iliac vein, both common iliac vein, as well as within the inferior vena cava, extending into the right atrium (Figure 4,5) as the features of echocardiography and abdominal ultrasound. Therefore, the presumptive diagnosis of sarcoma with invasion in right internal iliac vein, both common iliac vein, the inferior vena cava, as well as the right atrium was made by multi-detector-row computerized tomography.

The patient underwent a one-stage combined multidisciplinary including department of gynaecology, cardiac surgery and vascular surgery thoraco-abdominal

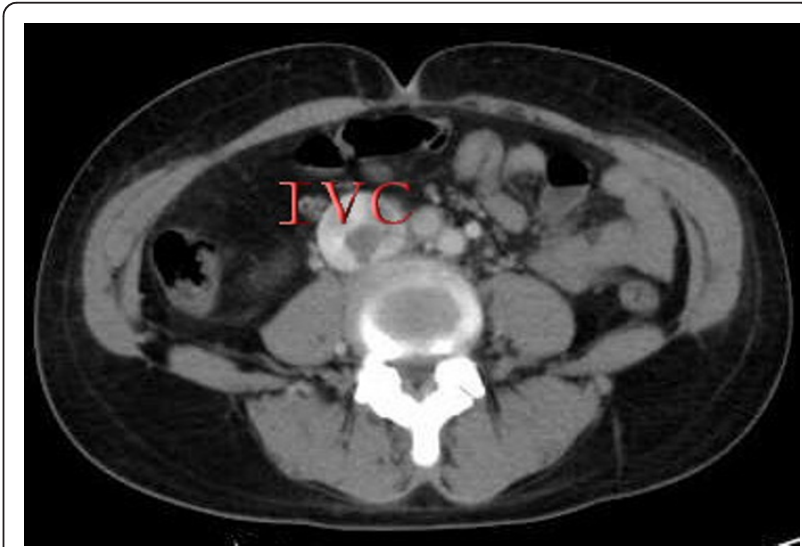

Figure 4 Abdominal computerized tomography revealed a low attenuation-filling continued defect within the inferior vena cava. IVC: inferior vena cava. 


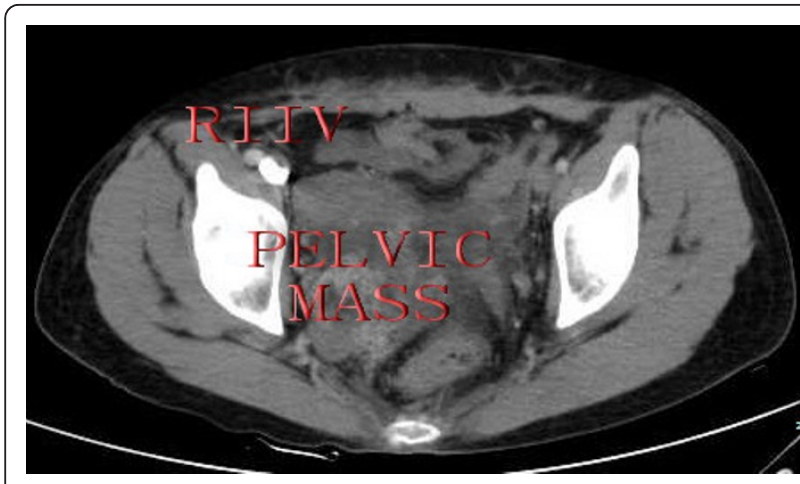

Figure 5 Pelvic computerized tomography revealed a lobulated pelvic mass, a low attenuation-filling continued defect within right internal iliac vein. RIIV: right internal iliac vein.

operation under general anaesthetic. The pelvic mass was excised by gynaecological team. The tumor within vascular and heart was upwardly dislodged by opening the right atrium. The length of tumor within vascular and heart was $25 \mathrm{~cm}$. Consistent with the features of echocardiography and abdominal ultrasound, the surgical views revealed that the tumor within vascular and heart has no stalk and has well-demarcated borders with the wall of vascular and heart. Subsequently the pathologic report confirmed IVL.

\section{Discussion}

IVL is histologically confirmed benign smooth muscle tumor within vascular spaces from intrauterine venules to the systemic veins including iliac vein, the inferior vena cava, even extending into the right heart chamber and pulmonary artery and its extrauterine involvement occurs in approximately $30 \%$ of cases and intracardiac extension accounts for about 10\% [4,5]. Birch-Hirschfeld [6] first presented case of IVL in 1896 and Durck [7] first presented case of intracardiac extension of IVL in 1907. Although the pathogenesis of IVL remain unclear, there are two main theories regarding the etiology of IVL. The first theory is that IVL is leiomyoma originated from the venous wall [8] and the other is that IVL is the uterine leiomyoma which invaded into the uterine vein [9]. In the present case there was evidence of uterine leiomyoma invading into the vessels provided by the feature of echocardiography, abdominal ultrasound and computerized tomography. As detected by echocardiography and abdominal and viewed by surgery, the intravascular and intraatrium tumor mass were not attached to the endothelial surface and endocardium but were freely movable within the vascular and heart spaces, which was easily removed. These features suggest that IVL may be the uterine leiomyoma which invaded into the uterine vein in the present cases. Up to date, the mechanisms of diffusion to the heart in IVL patients remain unclear. However, incomplete surgery hysterectomy has been presumed to promote the proliferation of IVL. In addition, Yaguchi $C$ et al [10] reported that hyaluronan, which is an important constituent of the extracellular matrix and is known to regulate cellular events, was expressed more prominently in IVL than in uterine leiomyomas by immunohistochemical studies and propose that IVL has viscoelastic properties and contains a large amount of hyaluronan, which may promote invasion during pathogenesis.

IVL is usually occurring in women of ages 23 to 80 (median age, 44 years) [11]. Most patients, including the present case, have a history of hysterectomy. It is presumed that IVL had simply not been diagnosed in the period of time immediately following the hysterectomy. A tumor left inside the small pelvic veins at the time of hysterectomy [12], which could not be detected by all kinds of imaging methods including ultrasound, computerized tomography and MRI etc., could have extended towards the right heart, leading to obstruction. Kuenen $\mathrm{BC}$ et al [13] reported that there was 4-year median interval from performing hysterectomy to the diagnosis of IVL with extension to heart and differences in cytological features only related to the time period of the interval from performing hysterectomy to the diagnosis of IVL with extension to heart [14]. Therefore, the present case could have more athletic cytological features.

The clinical characteristics of IVL are usually similar to typical uterine leiomyomas and relates to the obstructive effect of the tumor on the tricuspid orifice and disturbance of venous return, which could include dyspnea on exertion, syncopal episodes, pulmonary embolism, and sudden death [15-17]. The current patient presented with debilitation and engorgement of both lower extremities. The presence of debilitation and engorgement of both lower extremities motivated echocardiographic and abdominal ultrasonographic examination revealing the presence of a right heart chamber and the inferior vena cava tumor mass. To reveal the features of Chinese IVL patients, The clinical features, treatment of the IVL patients with extension to heart in China like the present case from 2001 to 2011 (in that studies all the patients have detailed clinical parameters) are summarized by exploring the Pubmed in Table 1 to display the features of Chinese IVL patients [18-20]. As same as the present case, the Chinese IVL patients usually have the history of hysterectomy.

Due to its rarity, the diagnosis of IVL is often overlooked. Echocardiography, abdominal ultrasound, computerized tomography and MRI etc. are available for the detection and diagnosis of IVL. When an abdominal pelvic intravenous mass is demonstrated, different diagnosis include abdominal tumors with metastasis to 
Table 1 The clinical features and treatment of Chinese IVL patients with extension to heart

\begin{tabular}{cccccc}
\hline Case & Age & Presentation & History of hysterectomy & Treatment & Follow-up without recurrence \\
\hline 1 & 52 & Shortness of breath and palpitation $^{18}$ & No data & Two-stage operation & Two-year \\
2 & 49 & Palpitation & \\
3 & 39 & Chest tightness and dyspnea & No data & Two-stage operation & Six-month \\
4 & 40 & Dyspnea on exertion and pedal edema ${ }^{20}$ & Yes & Two-stage operation & No data \\
\hline
\end{tabular}

the systemic vein, such as hepatocellular carcinoma, renal cell carcinoma, adrenal tumor and leiomyosarcoma of the uterus etc., bland thrombus, or leiomyosarcoma of the inferior vena cava [21,22]. The features and attachment site of the tumor detected by echocardiography, abdominal ultrasound, computerized tomography and MRI may offer important information regarding the nature of the tumor. The presence of a long, serpentine, polypoidal and elongated mobile mass extending from the inferior vena cava or the veins of the lower body into the right atrium should raise the suspicion of IVL $[23,24]$. Of course, direct connection between the intravenous mass and the uterus or adnexa, and absence of renal, hepatic or adrenal masses, favor the diagnosis of IVL. In the present case, ultrasound and computerized tomography revealed a long, serpentine, polypoidal and elongated mobile mass extending from right internal iliac vein continued to the inferior vena cava extending into the right atrium and the mass related to pelvic mass at absence of renal, hepatic or adrenal masses. So the ultrasonographer raised the presumptive diagnosis of IVL. To our knowledge, this is the first case which was preoperatively confirmed. Of course, the thrombus within right heart chamber atrium and inferior vena cava can also be mobile or free-floating. Contrast-enhanced computerized tomography or MRI can aid in the different diagnosis of IVL and thrombus.

To date, the complete surgical excision is the first and best treatment of choice for IVL, although antiestrogen therapy had also been proposed [25]. IVL usually adhere to but do not invade the vessel wall and therefore, can usually be removed by downward traction from the ovarian vein, iliac vein or inferior vena cava. However, a combined multidisciplinary thoraco-abdominal operation with removal cardiopulmonary bypass and the removal of tumour in the inferior vena cava and right heart chamber is indicated if IVL is extending to the right heart chamber. In the present case, the intra-cardiac and intra-vascular mass was free-floating without involvement of the cardiac structure and vein wall tissue. Therefore, surgery was performed by a one-stage operation, involving upward dislodgment of the intracardiac and intra-vascular tumor by opening the right atrium and resection of the pelvic tumor. Of course, the two-stage operation seems usually to be performed in Chinese patients with IVL and extension to heart and there are no recurrences in short period.

\section{Consent}

Written informed consent was obtained from the patient for publication of this case report and accompanying images. A copy of the written consent is available for review by the Editor-in-Chief of this journal.

\section{Acknowledgements}

Dr. Lou YF was supported by the Bureau of Traditional Chinese Medicine of Zhejiang (2008CB033) and health department of Zhejiang (2010KYA090). Dr. Song ZZ was supported by the Bureau of Traditional Chinese Medicine of Zhejiang (2009CB036) and health department of Zhejiang (2009B058).

\section{Author details}

${ }^{1}$ Department of Laboratory, The First Affiliated Hospital, College of Medicine, Zhejiang University, Hangzhou, China. ${ }^{2}$ Department of Laboratory, Tong-De Hospital of Zhejiang Province, Hangzhou, China. ${ }^{3}$ Department of Ultrasound, The First Affiliated Hospital, College of Medicine, Zhejiang University, Hangzhou, China.

\section{Authors' contributions}

YFL collected the related information, carried out laboratory examination and drafted the manuscript. ZZS carried out Echocardiographic examination and revised the manuscript. All authors read and approved the final manuscript.

\section{Competing interests}

The authors declare that they have no competing interests.

Received: 14 September 2011 Accepted: 24 September 2011 Published: 24 September 2011

\section{References}

1. Vaideeswar P, Kulkarni DV, Karunamurthy A, Hira P: Intracardiac leiomyomatosis: Report of two cases. Indian J Pathol Microbiol 2011, 54:158-160.

2. Kocica MJ, Vranes MR, Kostic D, Kovacevic-Kostic N, Lackovic V, BozicMihajlovic V, Velinovic MM, Mikic ADj, Dimitrijevic-Kalezic N: Intravenous leiomyomatosis with extension to the heart: rare or underestimated? J Thorac Cardiovasc Surg 2005, 130:1724-1726.

3. Timmis AD, Smallpeice C, Davies AC, Macarthur AM, Gishen P, Jackson G: Intracardiac spread of intravenous leiomyomatosis with successful surgical excision. N Engl J Med 1980, 303:1043-1044.

4. Mulvany NJ, Slavin JL, Ostor AG, Fortune DW: Intravenous leiomyomatosis of the uterus: A clinicopathologic study of 22 cases. Int J Gynecol Pathol 1994, 13:1-9.

5. Baca Lopez FM, Martınez-Enriquez A, Castrejon-Aivar FJ, Ruanova-León D, Yánez-Gutiérrez L: Echocardiographic study of an intravenous leiomyoma: Case report and review of the literature. Echocardiography 2003, 20:723-725.

6. Birch-Hirschfeld FV: Lehrbuch der pathologischen Anatomie. Leipzig, Germany: FCW Vogel; 5 1896, 266. 
7. Durck H: Ueber ien Kontinvierlich durch die entere Holhlvene in das Herz vorwachsendes: Fibromyom des uterus. Munchen Med wehnschr 1907, 54:1154.

8. Knauer E: Beitrag zur Anatomie der Uterusmyome. Beitr z Gynäk 1903, 1:695.

9. Sitzenfrey A: Ueber Venenmyome des Uterus mit intravaskulärem. Ztschr f Geburtsh u Gynäk 1911, 68:1.

10. Yaguchi C, Oi H, Kobayashi H, Miura K, Kanayama N: A case of intravenous leiomyomatosis with high levels of hyaluronan. J Obstet Gynaecol Res 2010, 36:454-458.

11. Clement PB: Intravenous leiomyomatosis of the uterus. Pathol Annu 1988, 23:153-183.

12. Du J, Zhao X, Guo D, Li H, Sun B: Intravenous leiomyomatosis of the uterus: A clinicopathologic study of 18 cases, with emphasis on early diagnosis and appropriate treatment strategies. Human pathology 2011, 42:1240-1246.

13. Kuenen BC, Slee PH, Seldenrijk CA, Wagenaar SS: Intravenous leiomyomatosis complicated by Budd-Chiari syndrome. Postgrad Med J 1996, 72:686-688.

14. Clement $\mathrm{PB}$, Young RH, Scully RE: Intravenous leiomyomatosis of the uterus. A clinicopathological analysis of 16 cases with unusual histologic features. Am J Surg Pathol 1998, 12:932-945.

15. Nam MS, Jeon MJ, Kim YT, Kim JW, Park KH, Hong YS: Pelvic leiomyomatosis with intracaval and intracardiac extension: A case report and review of the literature. Gynecol Oncol 2003, 89:175-180.

16. Marcus SG, Krauss T, Freedberg RS, Culliford AT, Weinreich DJ, Kronzon I: Pulmonary embolectomy for intravenous uterine leiomyomatosis. Am Heart J 1994, 127:1642-1645.

17. Roman DA, Mirchandani H: Intravenous leiomyomatosis with intracardiac extension causing sudden death. Arch Pathol Lab Med 1987, 111:1176-1178.

18. Yu L, Shi E, Gu T, Xiu Z, Fang Q, Wang C: Intravenous Leiomyomatosis with Intracardiac Extension: A Report of Two Cases. Journal of Cardiac Surgeny 2011, 26:56-60.

19. Wu CK, Luo JL, Yang CY, Huang YT, Wu XM, Cheng CL, Chiang FT, Tseng CD: Intravenous leiomyomatosis with intracardiac extension. Intern Med 2009, 48:997-1001

20. Fang $\mathrm{BR}, \mathrm{Ng} \mathrm{YT}$, Yeh $\mathrm{CH}$ : Intravenous leiomyomatosis with extension to the heart: echocardiographic features: a case report. Angiology 2007, 58:376-379.

21. Kato Y, Tanaka N, Kobayashi K, Ikeda T, Hattori N, Nonomura A: Growth of hepatocellular carcinoma into the right atrium: Report of five cases. Ann Intern Med 1983, 99:472-474.

22. Nesbitt JC, Soltero ER, Dinney CP, Walsh GL, Schrump DS, Swanson DA, Pisters LL, Willis KD, Putnam JB Jr: Surgical management of renal cell carcinoma with inferior vena cava tumor thrombus. Ann Thorac Surg 1997, 63:1592-1600.

23. Maurer G, Nanda NC: Two-dimensional echocardiographic identification of intracardiac leiomyomatosis. Am Heart J 1982, 103:915-917.

24. Kullo IJ, Oh JK, Keeney GL, Khandheria BK, Seward JB: Intracardiac leiomyomatosis: echocardiographic features. Chest 1999, 115:587-591.

25. Mitsuhashi A, Nagai Y, Sugita M, Nakajima N, Sekiya S: GnRH agonist for intravenous leiomyomatosis with cardiac extension. A case report J Reprod Med 1999, 44:883-886.

doi:10.1186/1476-7120-9-25

Cite this article as: Lou et al:: Intravenous leiomyomatosis of the uterus with extension to the right heart. Cardiovascular Ultrasound 2011 9:25.

\section{Submit your next manuscript to BioMed Central and take full advantage of:}

- Convenient online submission

- Thorough peer review

- No space constraints or color figure charges

- Immediate publication on acceptance

- Inclusion in PubMed, CAS, Scopus and Google Scholar

- Research which is freely available for redistribution 